\title{
Síndrome de Burnout em trabalhadores de uma empresa de base florestal com serviços de silvicultura
}

\author{
Burnout syndrome in workers of a forest-based company with forestry services
}

Síndrome de Burnout en trabajadores de una empresa de base forestal con servicios de silvicultura

Carla Lorena de Araujo Costa ${ }^{1 *}$, Robinson Magalhães Maia², Marcos Lázaro da Silva Guerreiro $^{3}$, Rodolfo Bello Exler ${ }^{4}$.

\section{RESUMO}

Objetivo: Identificar a presença da Síndrome de Burnout entre os silvicultores de uma empresa de base florestal. Métodos: Trata-se de uma pesquisa de estudo de coorte transversal, descritiva e de base populacional utilizando como instrumentos o Maslach Burnout Inventory (MBI), traduzido e validado para o Brasil. A amostra foi composta por 26 silvicultores florestais e a coleta de dados realizada no mês de agosto de 2017, com entrevistas face a face. O banco de dados foi criado e analisado através do programa computacional Microsoft Office Excel 2007. Resultados: Dos 26 trabalhadores florestais, 50\% apresentava nível moderado de exaustão emocional, 35\% cursavam com moderado nível de despersonalização e apenas $12 \%$ evoluiu com nível baixo de reduzida realização profissional; em relação à presença da síndrome, $58 \%$ encontra-se em nível leve e $23 \%$ em nível moderado. Conclusão: Constatou-se que o Burnout se encontra presente na vida de alguns silvicultores, sendo premente a necessidade urgente de elaboração e implantação de estratégias que possam amenizar a sobrecarga de trabalho nos indivíduos susceptíveis e evitar agravos físicos e mentais para saúde dos mesmos.

Palavras-chave: Esgotamento Profissional, Eucalyptus, Silvicultura.

\begin{abstract}
Objective: To identify the presence of Burnout Syndrome among foresters of a forest-based company. Methods: This is a cross-sectional, descriptive and population-based cohort study using the Maslach Burnout Inventory (MBI), translated and validated for Brazil. The sample consisted of 26 forest foresters and the data collection performed in August 2017, with face-to-face interviews. Results: Of the 26 forest workers, $50 \%$ had a moderate level of emotional exhaustion, 35\% had a moderate level of depersonalization, and only $12 \%$ had a low level of emotional exhaustion. reduced professional achievement; in relation to the presence of the syndrome, $58 \%$ is at a mild level and $23 \%$ at a moderate level. Conclusion: It was verified that Burnout is present in the lives of some foresters, being urgent the urgent need of elaboration and implementation of strategies that can reduce the work overload in the susceptible individuals and avoid physical and mental damages to their health.
\end{abstract}

Keywords: Professional Exhaustion, Eucalyptus, Forestry.

${ }^{1}$ Docente da Faculdade Unirb Alagoinha-BA e Faculdade Santo Antônio de Alagoinhas-BA.

*E-mail: c_lorenacosta@yahoo.com.br

${ }^{2}$ Docente do Programa de Mestrado Profissional em tecnologias aplicáveis à Bioenergia da Faculdade de Tecnologia e Ciências em Salvador-BA.

${ }^{3}$ Docente do quadro permanente do Mestrado Profissional em tecnologias aplicáveis à Bioenergia da Faculdade de Tecnologia e Ciências em Salvador-BA.

${ }^{4}$ Docente do Centro Universitário Senai Cimatec, Salvador-BA.

SUBMETIDO EM: 3/2019

ACEITO EM: 4/2019

PUBLICADO EM: 6/2019

REAS/EJCH | Vol. 11 (11) | e590 | DOI: https://doi.org/10.25248/reas.e590.2019 Página 1 de 7 


\section{RESUMEN}

Objetivo: Identificar la presencia del Síndrome de Burnout entre los silvicultores de una empresa de base forestal. Métodos: Se trata de una encuesta de cohorte transversal, descriptiva y de base poblacional, utilizando como instrumentos el Maslach Burnout Inventory (MBI), traducido y validado para Brasil. La muestra fue compuesta por 26 silvicultores forestales y la recolección de datos realizada en el mes de agosto de 2017, con entrevistas cara a cara. La base de datos fue creada y analizada a través del programa computacional Microsoft Office Excel 2007. Resultados: De los 26 trabajadores forestales, el 50\% presentaba un nivel moderado de agotamiento emocional, el $35 \%$ cursaba con moderado nivel de despersonalización y sólo el $12 \%$ evolucionó con un nivel bajo reducida realización profesional; en relación a la presencia del síndrome, el $58 \%$ se encuentra a nivel leve y el $23 \%$ a nivel moderado. Conclusión: Se constató que el Burnout se encuentra presente en la vida de algunos silvicultores, siendo urgente la necesidad urgente de elaboración e implantación de estrategias que puedan amenizar la sobrecarga de trabajo en los individuos susceptibles y evitar agravios físicos y mentales para la salud de los mismos.

Palabras clave: Agotamiento Profesional, Eucalyptus, Silvicultura.

\section{INTRODUÇÃO}

O Brasil apresenta grande potencial para utilização de matrizes energéticas renováveis (COSTA RC e PRATES CP, 2005) na posição de um dos maiores produtores agrícolas e florestais do mundo (DIAS et al., 2012). As plantações florestais, além de fornecerem matéria-prima para os diversos usos industriais e domésticos, cumprem diversas funções ambientais e sociais, desempenham um papel importante no desenvolvimento sustentável e contribuem com a conservação das florestas nativas (LIMA CGR, et al., 2010).

Atualmente, as espécies do gênero Eucalyptus são as mais utilizadas para a produção de carvão vegetal, suas características de rápido crescimento e densidade consideráveis garantem a produção de um carvão facilmente renovável e de boa qualidade. Contudo, para que haja a utilização da matéria-prima de eucalipto é preciso que ocorra atividade florestal (CASTRO AFNM, 2011)

Conforme Cordeiro R et al. (2005) durante o manejo florestal, tanto exploração quanto a colheita florestal são serviços referentes ao corte (derrubada) da madeira é realizado por trabalhadores que se expõem a diversas condições laborais. Os trabalhadores que realizam o corte de eucalipto estão expostos constantemente a diversas situações de risco durante o desempenho de suas atividades, devido ao uso incessante de motosserras e maquinários pesados.

A depender da maneira como as atividades florestais são executadas, os trabalhadores podem desenvolver doenças ocupacionais que conduzem a desordens físicas, psíquicas e sociais e consequentemente ao desenvolvimento de doenças ocupacionais. Neste sentido, uma das doenças ocupacionais atualmente em destaque é a síndrome de Burnout (SB) ou síndrome do esgotamento profissional (FILDER NC, et al., 2003).

Segundo Murofuse NT et al. (2005) a Burnout é um distúrbio psíquico, descrito em 1974 por um médico americano chamado Freudenberger. A principal característica da síndrome é o estado de tensão emocional e estresse crônico provocado por condições de trabalho físicas, emocionais e psicológicas desgastantes. $O$ sintoma típico da síndrome é a sensação de esgotamento físico e emocional que se reflete em atitudes negativas, absenteísmo, presenteísmo, agressividade, depressão, baixa autoestima, dentre outros.

A síndrome ainda é pouco conhecida pelos trabalhadores e pelos profissionais da previdência social, trata-se, portanto, de uma doença pouco difundida pela mídia, bem como no meio acadêmico. Os trabalhadores que fazem parte do grupo de risco não têm conhecimento a respeito da Burnout e nem tampouco dos fatores que poderão facilitar o aparecimento da síndrome. Indivíduos que trabalham em empresas de base florestal com serviços de silvicultura estão expostos a constantes agentes causadores de 
doenças laborais, tornando-os assim, parte integrante do grupo de risco para o desenvolvimento da síndrome de Burnout (SANTOS JD e NASCIMENTO AF, 2015).

Neste sentido, o presente trabalho se propôs a identificar a síndrome de Burnout em funcionários de uma empresa de base florestal com serviços de silvicultura. Os dados obtidos poderão ser utilizados como referencial para auxílio na compreensão da síndrome em questão, fornecendo elementos que servirão de alerta e reflexão, tanto para os que planejam e administram empresas de silvicultura, como para aqueles que executam o trabalho diariamente.

\section{MÉTODOS}

Trata-se de um estudo de coorte transversal, caráter descritivo e de base populacional, referente a um grupo de trabalhadores que prestam serviço de silvicultura na Bahia, que tinham experiência laboral com o corte do eucalipto e que aceitaram participar do estudo, assinando o termo de consentimento livre e esclarecido (TCLE).

Foram utilizados como critério de inclusão, funcionários ativos no setor e como critério de exclusão aqueles que ocupam o cargo há menos de dois meses, estando assim em período de adaptação ao setor.

O instrumento de pesquisa foi composto pelas condições sociodemográficas, laborais, de saúde e estilo de vida. Para avaliar a presença da SB, utilizou-se o Maslach Burnoutt Inventtory (MBI). No momento em que houve a convocação para participar da avaliação que indicava os traços da Síndrome de Burnout, alguns silvicultores ficaram inquietos e temerosos.

Inquietos por não saberem como lidar com o problema psíquico caso já estivesse instalado, temerosos em identificar uma doença incapacitante e que pudesse colocar em risco seus empregos. Contudo, a aplicabilidade do Questionário Preliminar de Identificação da Burnout elaborado e adaptado por Chafic Jbeili (inspirado no Maslach Burnout Inventory-MBI) ocorreu de forma tranquila e satisfatória.

O questionário (MBI) foi elaborado por Maslach e Jackson em 1981, porém validado no Brasil somente em 2001, onde foi possível identificar a síndrome de Burnout na população em estudo. Esse instrumento possui 20 itens e é o mais utilizado para mensurar o Burnout que avalia as três dimensões da síndrome (cansaço emocional, despersonalização e realização profissional) Cada item do inventário (MIB) apresenta cinco opções de resposta sendo "um" para "nunca", "dois" para "anualmente", "três" para "mensalmente", "quatro" para "semanalmente" e "cinco" para "diariamente". O escore do sujeito em cada uma das dimensões é calculado pelo somatório dos pontos dos itens relativos a cada uma das dimensões.

A aplicação dos questionários aos silvicultores ocorreu duas horas antes do início do expediente, no próprio local em que a empresa de base florestal presta serviços e logo após a entrevista, com os silvicultores já em campo, foi possível observar a rotina e o ambiente laboral dos mesmos. As análises dos dados estatísticos sucederam por meio da descrição das variáveis do estudo, através do programa computacional Microsoft Office Excel 2007.

Foram respeitados os aspectos éticos, seguindo a Resolução no 466/12 do Conselho Nacional de Saúde (CNS) por se tratar de pesquisa com seres humanos, tendo submetido o projeto ao Comitê de Ética da Faculdade de Tecnologia e Ciências da cidade de Salvador-Bahia, com parecer número CAAE 67845017.4.0000.5032.

Todos os participantes do estudo podem ter acesso em qualquer tempo às informações sobre os procedimentos, bem como benefícios e riscos relacionados à pesquisa, inclusive para dirimir eventuais dúvidas.

\section{RESULTADOS}

A população estudada foi composta por 26 trabalhadores florestais (100\%), com idade média de 36 anos, sendo a maioria dos indivíduos casada $(53,8 \%)$ e $77 \%$ possui filhos. Em relação à escolaridade aproximadamente $12 \%$ é de analfabetos e $38,47 \%$ apresentam o 1ํ grau incompleto. 
Na função ocupacional, 50\% é constituída por ajudante de operador de motosserra $31 \%$ operador de motosserra e 19\% operador de máquinas; o salário varia em função da ocupação, onde o operador de motosserra e seu ajudante, ganham um salário-mínimo (81\%).

Quanto ao período de trabalho e vínculo empregatício, respectivamente, 50\% encontra-se atuando há menos de 2 anos na empresa, contudo 100\% em situação efetiva em que todos os entrevistados têm a carteira profissional devidamente registrada e assinada pela empresa alvo da pesquisa.

Ao distribuir os níveis de cada dimensão do $\mathrm{MBI}$ entre os silvicultores em estudo, pôde-se observar que $50 \%$ apresentou nível moderado de exaustão emocional (EE); $65 \%$ apresentou nível baixo de despersonalização (DP); e na reduzida realização profissional (RRP), apenas $12 \%$ apresentou nível baixo (Tabela 1).

Tabela 1 - Distribuição dos resultados Maslach Burnoutt Inventtory (MBI) entre os funcionários de uma empresa de base florestal com serviços de silvicultura. ( $N=26)$

\begin{tabular}{l|c|c|c}
\hline \multicolumn{1}{c|}{ Dimensões } & \multicolumn{3}{c}{ Níveis N (\%) } \\
\cline { 2 - 4 } & Baixo & Moderado & Alto \\
\hline Exaustão Emocional & $05(19 \%)$ & $13(50 \%)$ & $08(31 \%)$ \\
\hline Despersonalização & $17(65 \%)$ & $09(35 \%)$ & 0 \\
\hline Reduzida Realização profissional & $03(12 \%)$ & $06(23 \%)$ & $17(65 \%)$ \\
\hline
\end{tabular}

Fonte: Autores (2019).

Sobre a realidade da Síndrome de Burnout (SB) entre os trabalhadores florestais em uma empresa de silvicultura, pôde-se constatar que dos 26 silvicultores entrevistados, $06(23 \%)$ a síndrome já começou a se instalar, $15(58 \%)$ encontra-se na fase inicial da síndrome e apenas $05(19 \%)$ apresenta possibilidade de desenvolver a SB (Gráfico 1).

Gráfico 1 - Realidade da Síndrome de Burnout entre funcionários de uma empresa de base florestal com serviços de silvicultura.

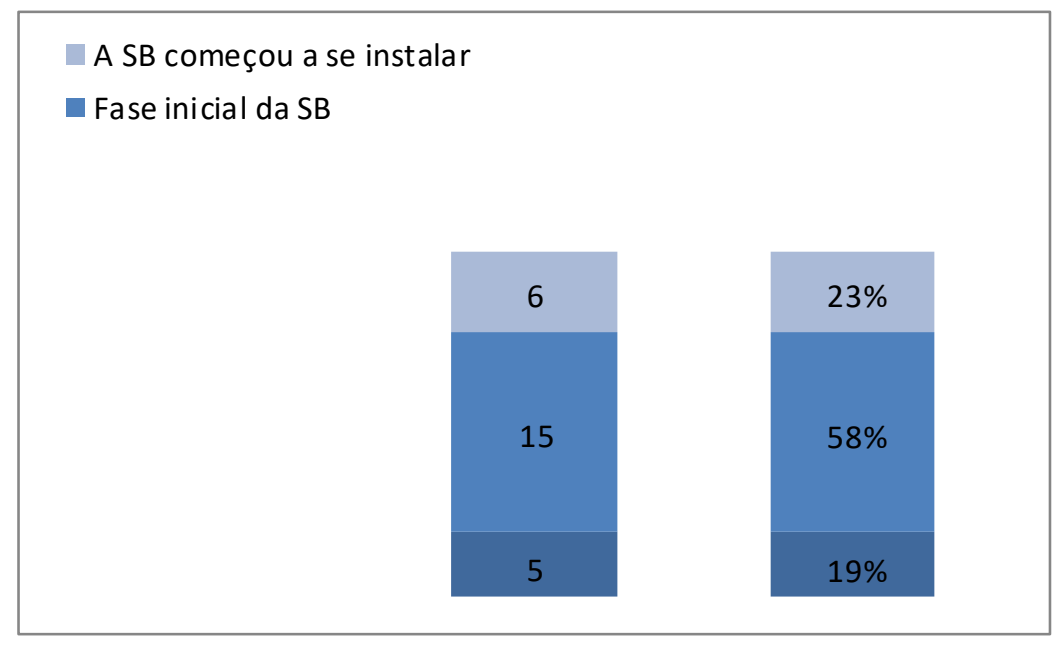

Fonte: Autores (2019)

\section{DISCUSSÃO}

A totalidade dos operadores envolvidos nesta pesquisa foi do sexo masculino, isso se deve ao estilo de trabalho realizado. Santos JD e Nascimento AF (2015) e Rodrigues CK et al. (2011), ambos concordam com o achado em relação ao predomínio do sexo masculino na atividade laboral de silvicultura. 
Em relação à idade média encontrada e ao grau de escolaridade, Silva EP et al. (2010) confirmam os dados obtidos nesse trabalho, quando descrevem em seu estudo que a média de idade foi em torno dos 36 anos, corroborando em relação à predominância da baixa escolaridade entre os trabalhadores florestais, já que, normalmente, esse tipo de trabalho é executado por pessoas com baixo grau de instrução, acostumados às condições de trabalho severas no campo e sem maiores oportunidades de inclusão no mercado de trabalho.

Quanto à profissão exercida, os estudos de Britto PC et al. (2015) mostram haver uma tendência de permanência dos trabalhadores na mesma função de ajudante de operador, operador de motosserra e operador de máquinas por não conseguirem visualizar uma ascensão profissional.

No que se refere à variação do salário, Ramos W et al. (2015) e Pieroni GB (2014) afirmaram em seus estudos que a remuneração do operador de motosserra varia no máximo de 1,5 salários-mínimos e que a remuneração salarial ocorre de acordo ao cargo e atividades exercidas.

Tendo em consideração sobre o período de trabalho e ao vínculo empregatício, Simões MRL e Rocha AM (2014), conseguiram observar o tempo de trabalho desses indivíduos onde média foi de 15 meses, com extremos que variaram de 3 meses a 27 anos de tempo de empresa. Contatando-se assim que grande parcela da população (44\%) era relativamente nova na empresa. Resultados idênticos, com relação ao registro em carteira profissional, foram encontrados por Rocha MB (2016), assim significa dizer que todos estão amparados pela legislação trabalhista e isso demonstra baixa rotatividade desse profissional na empresa.

Sobre as dimensões do MIB, a exaustão emocional (EE) é a primeira reação ao estresse gerado pelas exigências do trabalho, sendo considerada a característica central do Burnout, a manifestação mais óbvia, a principal queixa dos indivíduos que sofrem dessa síndrome (BATISTA JBV, et al., 2010). A explicação para $50 \%$ dos silvicultores apresentarem moderado nível de exaustão emocional pode estar parcialmente atribuída à atividade desenvolvida no setor florestal, uma vez que as funções, operadores de máquinas ou motosserra e ajudantes florestais, exigem demandas físicas que compõem um universo de trabalho desfavorável à saúde.

Albuquerque FJB (2012), ao averiguarem a presença de cansaço ao final de uma jornada de trabalho, mostraram que a presença de exaustão emocional relacionada ao cansaço excessivo em profissionais da Estratégia de Saúde da Família, uma vez que isto tem relação às limitações técnicas pessoais, materiais, elevada demanda de atendimentos, ao desrespeito de alguns usuários insatisfeitos e baixa remuneração. Algo similar foi notado em um estudo Truzzi A et al. (2012), com cuidadores familiares de pacientes com demência, que mostrou que $42,1 \%$ apresentou sentimentos de cansaço ao final de uma jornada de trabalho, principalmente, por conta dos altos níveis de ansiedade e declínio funcional dos doentes, associando a isto, quadros de depressão, fadiga, irritabilidade e diminuição do entusiasmo.

A segunda dimensão do MIB é a despersonalização profissional (DP) é uma forma de defesa da síndrome de Burnout. Revela-se por meio de atitudes de distanciamento emocional em relação às pessoas com as quais o trabalhador deve prestar seus serviços normais e com colegas de trabalho também. Os contatos tornam-se impessoais, desprovidos de afetividade, desumanos. Por vezes o indivíduo passa a apresentar comportamentos ríspidos, cínicos ou irônicos (BATISTA JBV, et al., 2010).

A baixa DP encontrada na amostra pode ser parcialmente justificada pelo simples fato de os funcionários da empresa, possuírem baixa escolaridade, uma vez que todos os trabalhadores encontram-se em situação efetiva com carteira profissional devidamente registrada e assinada pela empresa alvo da pesquisa e ganhando um valor fixo e diferenciado a depender da ocupação; além de um determinado quantitativo de trabalhadores que atuam há menos de 2 anos na empresa. Segundo Rocha MB (2016) todas essas projeções profissionais tornam as relações sociais menos conflituosas no ambiente laboral dificultando assim o adoecimento, principalmente a alterações de personalidade e resistência aos colegas do ambiente de trabalho. 
A última dimensão é a reduzida realização do trabalho (RRP), onde a execução dos afazeres ocupacionais decresce e o indivíduo perde a satisfação e a eficiência no trabalho. Há um sentimento de descontentamento pessoal, o laboral perde o sentido e passa a ser um fardo (BATISTA JBV, et al., 2010). Em relação à amostra da pesquisa, poucos entrevistados apresentaram baixo nível de RRP, possivelmente em razão do alto incide de satisfação profissional, relatado pelo os mesmos, uma vez que recebem um salário fixo e apresentam carteira assinada.

Os autores Jeong GJY e Kurcgant P (2010) resgataram como fatores de insatisfação, tanto aspectos pessoais, aflorando sentimentos relacionados ao significado do trabalho e ao modo de execução, como organizacionais, que dizem respeito à sobrecarga das atividades desenvolvidas e ao salário não compatível com a responsabilidade, levando à tristeza. Diante disto, observa-se que o grau de satisfação e motivação de uma pessoa, no que tange às condições de vida e trabalho, são fatores que podem abalar o equilíbrio e a estabilidade psicológica dentro do ambiente laboral, repercutindo na sua qualidade vida, levando à tristeza e melancolia prolongadas, interferindo no processo saúde-doença.

Conforme Benevides P (2014) após análise das dimensões, contidas no MIB, é preciso realizar o escore do sujeito em cada uma das dimensões, onde o cálculo é realizado através do somatório dos pontos dos itens relativos a cada uma das dimensões. As pontuações específicas identificam se o individuo apresenta desde nenhum indício da síndrome, até mesmo se já está em uma fase considerável da Burnout.

No presente estudo, observou-se que $58 \%$ dos silvicultores da amostra, encontram-se com nível leve da síndrome, enquanto $23 \%$ já estavam em nível moderado, contudo observou-se que os $19 \%$ não estão isentos de adquirir a Burnout, caso não haja uma intervenção por parte da empresa. Conceição ACR e Amorim AM (2015), ao analisarem a prevalência dos sinais e sintomas relacionados à síndrome de Burnout em trabalhadores do corte de cana-de-açúcar, observaram que $46,7 \%$ dos cortadores de cana apresentavam nível elevado de exaustão emocional, 47,2 \% cursavam com elevada despersonalização e $80,6 \%$ evoluíram com nível alto de insatisfação pessoal. Constatando assim que a prevalência, de níveis elevados da síndrome de Burnout entre os trabalhadores do corte da cana-de-açúcar, do município de Amélia Rodrigues, Bahia, foi de 9,4\%.

Santos e Nascimento (2015) aplicou o MIB nos silvicultores de eucalipto credenciados ao sindicato dos trabalhadores florestais, para identificar a existência do quadro de esgotamento profissional através da sintomatologia da SB. Apenas um participante da pesquisa foi observado e acompanhado. Após os resultados coletados, o trabalhador foi informado que estava na fase inicial da síndrome e resolveu procurar ajuda profissional.

\section{CONCLUSÃO}

A partir dos resultados apresentados, ficou evidente que a SB já estava presente na vida de alguns funcionários de uma empresa de base florestal com serviços de silvicultura. Constatou-se que amostra estudada se encontrava em um nível leve/moderado da patologia, medidas preventivas precisam, portanto, ser tomadas para impedir aumento do número de trabalhadores acometidos pela SB. Para os acometidos pela Burnout cabe à empresa identificá-los e encaminhá-los a um profissional que possa ajudar na reversão do quadro. Sem dúvida, estes resultados podem ser traduzidos em prejuízos tanto para a empresa, quanto para os trabalhadores envolvidos no processo. Assim sendo, é preciso que empreendimento compreenda a necessidade de urgência na elaboração e implantação de estratégias que possam amenizar a sobrecarga de trabalho e evitar os agravos físicos e mentais trazidos relacionados à atividade desenvolvida, além do fortalecimento de ações específicas de conscientização sobre o estresse laboral junto aos silvicultores da amostra, para que não se tornar crônico.

\section{REFERÊNCIAS}

1. ALBUQUERQUE FJB, et al. Avaliação da síndrome de burnout em profissionais da Estratégia Saúde da Família da capital paraibana. Psicologia: Reflexão e Crítica, 2012; 25 (3): 542-549. 
2. BATISTA JBV, et al. Prevalência da síndrome de burnout e fatores sociodemográficos e laborais em professores de escolas municipais da cidade de João Pessoa, PB. Revista Brasileira de Epidemiologia, 2010; 13 (3): 502-512.

3. BENEVIDES P (Org.). Burnout: quando o trabalho ameaça o bem-estar do trabalhador. São Paulo: Casa do Psicólogo; 2014; 21-91.

4. BRITTO PC, et al. Fatores humanos e condições de trabalho em atividades de implantação e manutenção florestal. Floresta e ambiente, 2015; 22 (4): 503- 512.

5. CASTRO AFNM. Efeito da idade e de materiais genéticos de Eucalyptus sp. na madeira e carvão de vegetal, Viçosa. Dissertação (Mestrado em Ciência Florestal) - Universidade Federal de Viçosa, Viçosa, 2011, 86p.

6. CONCEIÇÃO ACR, AMORIM AM. Sinais e sintomas relacionados a síndrome de Burnout em cortadores de cana-de-açúcar no município de Amélia Rodrigues- BA no ano de 2014. Dissertação (Mestrado em Mestrado em Bioenergia) - Instituto Mantenedor de Ensino Superior da Bahia, Salvador, 2015, 86p.

7. CORDEIRO R, et al. Exposição ao ruído ocupacional como fator de risco para acidentes do trabalho. Rev Saúde Pública, 2005; 39 (3):461-466.

8. COSTA RC, PRATES CP. O papel das fontes renováveis de energia no desenvolvimento do setor energético e barreiras à sua penetração no mercado. BNDES Setorial. Rio de Janeiro, 2005; 21: 5-30.

9. DIAS MOS, et al. Production of bioethanol and other bio-based materials from sugarcane bagasse: Integration to conventional bioethanol production process. Chemical Engineering Research \& Design, 2012; 87: 12061216.

10. FIEDLER NC, et al. Avaliação biomecânica dos trabalhadores em marcenarias no Distrito Federal. Ciência Florestal, 2003; 13: 99-109.

11. JEONG DJY, KURCGANT P. Factors of work dissatisfaction according to the perception of nurses of a university hospital. Revista Gaúcha de Enfermagem, 2010; 31 (4): 655-661.

12. LIMA CGR, et al. Atributos físico-químicos de um Latossolo do cerrado brasileiro e sua relação com características dendrométricas do eucalipto. Revista Brasileira de Ciência do Solo, 2010; 34(1): 163-173.

13. MUROFUSE NT, et al. Reflexões sobre Estresse e Burnout e a relação com a enfermagem. Rev. Latino-am. Enfermagem, 2005; 13 (2): 225-261.

14. PIERONI GB. Análise ergonômica do trabalho florestal em uma empresa de produção de madeira em toras. Dissertação (Monografia de especialização). Universidade Tecnológica Federal do Paraná, Pato Branco - PR, 2014, 65p.

15. RAMOS W, et al. Análise de fatores humanos na colheita florestal com motossera: um estudo na região de Jaguariaíve- PA. XXXV Encontro NacionaL de Engenharia de Produção. Perspectivas Globais para a Engenharia de Produção Fortaleza, CE, Brasil, 2015.

16. ROCHA MB. Fatores e riscos ergonômicos na colheita florestal mecanizada. Dissertação (Mestrado em ciências Florestais). Universidade Estadual do Sudoeste da Bahia, Vitória da Conquista, 2016, 55p.

17. RODRIGUES CK, et al. Avaliação de risco de Ler/ Dort nas atividades de roçado manual e semimecanizada em povoamentos florestais. Rev. Recursos Florestais e Engenharia Florestal, 2011; 58 (5): 584-592.

18. SANTOS JD, NASCIMENTO AF. O sofrimento no trabalho e a síndrome de burnout nos trabalhadores das fazendas de eucalipto na região de Alagoinhas- Bahia. Bioenergia: Um Diálogo Renovável, 2015; 4:81- 92.

19. SILVA EP, et al. Diagnóstico das condições de saúde de trabalhadores envolvidos na atividade em extração manual de madeira. Revista Árvore, 2010; 34 (3): 561-565.

20. SIMÕES MRL, ROCHA AM. Absenteísmo-doença entre trabalhadores de uma empresa florestal no Estado de Minas Gerais, Brasil. Revista Brasileira de Saúde Ocupacional, 2014; 39 (129): 17-25.

21. TRUZZI A, et al. Burnout in Familial Caregivers of Patients with Dementia. Revista Brasileira de Psiquiatria, 2012; 34 (4): 405-412. 